\title{
A scalable fabrication process of polymer microneedles
}

\author{
This article was published in the following Dove Press journal: \\ International Journal of Nanomedicine \\ 9 March 2012 \\ Number of times this article has been viewed
}

\section{Sixing Yang \\ Yan Feng \\ Lijun Zhang \\ Nixiang Chen \\ Weien Yuan \\ Tuo Jin}

School of Pharmacy, Shanghai Jiao Tong University, Shanghai, People's

Republic of China
Correspondence: Tuo Jin, Weien Yuan School of Pharmacy, Shanghai Jiao

Tong University, 800 Dongchuan Road, Shanghai 200240,

People's Republic of China

Tel +86 2I 34204695

Fax +86 2I 34205072

Email tjin@situ.edu.cn;

yuanweien@I26.com
Abstract: While polymer microneedles may easily be fabricated by casting a solution in a mold, either centrifugation or vacuumizing is needed to pull the viscous polymer solution into the microholes of the mold. We report a novel process to fabricate polymer microneedles with a one-sided vacuum using a ceramic mold that is breathable but water impermeable. A polymer solution containing polyvinyl alcohol and polysaccharide was cast in a ceramic mold and then pulled into the microholes by a vacuum applied to the opposite side of the mold. After cross-linking and solidification through freeze-thawing, the microneedle patch was detached from the mold and transferred with a specially designed instrument for the drying process, during which the patch shrank evenly to form an array of regular and uniform needles without deformation. Moreover, the shrinkage of the patches helped to reduce the needles' size to ease microfabrication of the male mold. The dried microneedle patches were finally punched to the desired sizes to achieve various properties, including sufficient strength to penetrate skin, microneedles-absorbed water-swelling ratios, and drug-release kinetics. The results showed that the microneedles were strong enough to penetrate pigskin and that their performance was satisfactory in terms of swelling and drug release.

Keywords: polymer microneedles, ceramic mold, polyvinyl alcohol, swelling

\section{Introduction}

Microneedle technology has been developing as a drug-delivery system for more than a decade. ${ }^{1}$ The principal advantage of microneedles is that they can provide a minimally invasive means of transporting molecules into the skin. ${ }^{2}$ This is particularly true for protein and peptide drugs, which are mostly administered by frequent injections due to their large molecular weight, short in vivo life, and poor tissue membrane permeability. ${ }^{3}$ Frequent injections are commonly used for many protein/peptide drugs in the treatment of chronic conditions, eg, insulin for diabetes and calcitonin for osteoporosis. Guided by this goal, practitioners have employed a number of specific strategies to use microneedles for transdermal delivery. Based on their structures and materials, microneedles may be classified into three categories: inorganic solid microneedles, ${ }^{4,5}$ hollow microneedles, ${ }^{6,7}$ and polymer microneedles. ${ }^{8-10}$ Among these microneedle technologies, polymer microneedles are the most feasible due to their uncomplicated fabrication and low cost.

Molding has been commonly used in the fabrication of polymer microneedles. ${ }^{8,11,12}$ In brief, the drug-loaded polymer solution is pulled into microholes after casting on the surface of the female microneedle mold, which is made of polydimethylsiloxane. ${ }^{8,11-13}$ Methods reported in achieving this process include centrifugation ${ }^{8}$ and vacuumizing, ${ }^{11,12}$ 
in which the bubbles in the microholes are exhausted to the surface of the polymer solution. However, centrifugation is restricted in its use due to size limitations of centrifuge rotors. In addition, polymer solutions used with a vacuum must be prepared in a low concentration or viscosity, which is a complicated operation. Furthermore, the low consistency of the polymer can make the drying process challenging, since deformation occurs when water is removed. Sullivan ${ }^{11}$ prepared microneedles with polymerization of liquid monomer, which showed satisfactory fluidity. However, the introduction of a chemical initiator may damage the protein drug's activity as well as human skin in treatment.

In this study, we report a novel fabrication method of polymer microneedles to solve the problems mentioned above. Chinese purple sand was used to prepare the female mold. Purple sand is a material that is commonly used in the production of traditional Chinese ceramic teapots and cups, ${ }^{14}$ and it has no adverse effects on the human body. ${ }^{15}$ Besides its durability and characteristic hardness preventing deformation, the main reason for choosing purple sand as a female mold was its property of being breathable but water impermeable. The breathable property was used to pull the polymer solution into the microholes, with a vacuum under the female mold (Figure 1) to simplify the operation. The water impermeability was useful in preventing drug loss due to siphonage. With this female mold, we fabricated a microneedle patch using polyvinyl alcohol (PVA) solution, which was cross-linked and solidified by freeze-thawing to form microneedles. Free drying and fixed drying were used as two separate steps in the drying process. Free drying was used to adjust the intervals of the microneedles because of shrinking. This drying step solved the limitation of the size of the microholes in the mold, thus reducing the difficulty and cost of mold preparation. Fixed drying was used to remove the remaining water and make the needles sufficiently hard. Integrative and partial drug-loaded microneedles were fabricated to ensure sustained release and fast-acting treatment, respectively (Figure 1).

\section{Materials and methods \\ Materials}

The purple sand was provided by Ceramics Research Institute (Yixing, China). The PVA, dextran, and sodium carboxymethylcellulose (CMC) used in this study were purchased from Sigma Corporation (St Louis, MO).

\section{Molding}

Thousands of hexagonal steel sticks were finished into needles with a tip angle of $\alpha$ and then cut into the same length $(5 \mathrm{~cm})$, as shown in Figure 2A. All needles were gathered and fixed in a steel bucket, followed by the nesting of a Teflon board. The board was made with an array of microholes to meet the needles' tips. After fixing the board with screws, the core of the male mold was prepared (Figure 2B). According to the core's size, a stainless shell and lid were prepared to form space for the purple sand. The shell was a combination of two semicolumns (Figure 2C), which could be separated when detaching the purple-sand mold.

Purple-sand mud was firstly prepared by blending dry purple sand and water in a ratio of 50:9. Forty-five grams of mud (with a particle size of $1-5 \mu \mathrm{m}$ ) was smoothly dispersed into the male mold, followed by a pressing of the lid at 400 psi with a hand-operated hydraulic press (YS20; Qirui Tech, Suzhou, China). After separation from the shell, the purplesand mold was placed on an experiment table for 12 hours to air-dry. This adobe was transferred into an electric furnace

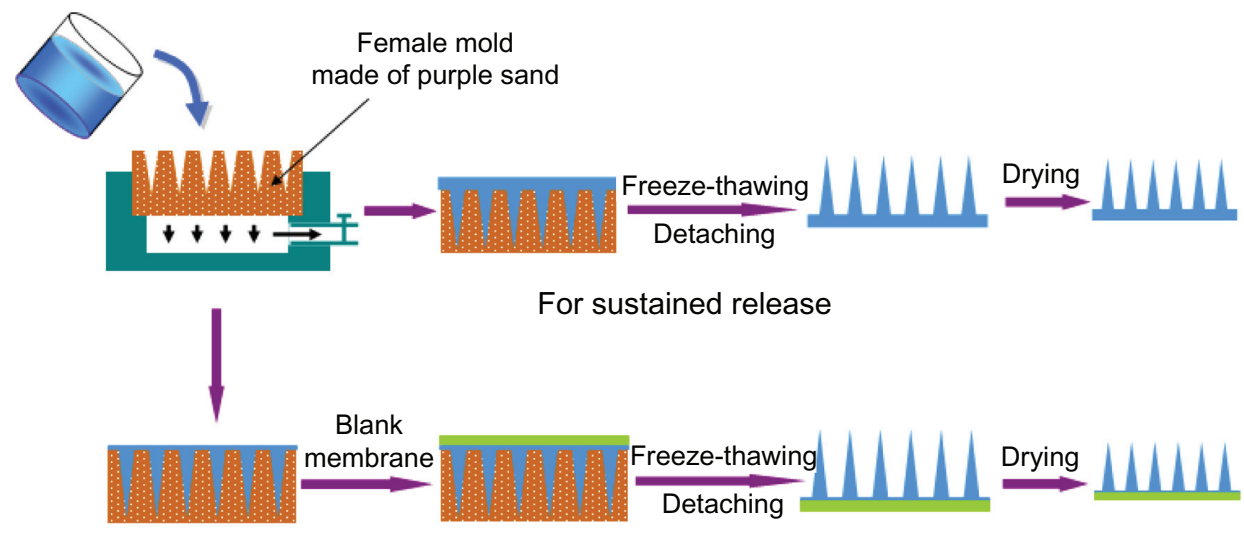

For fast acting

Figure I Full view of fabrication process of polymer microneedle patch. The patch was fabricated by casting polymer solution in mold, cross-linking to form needles through freeze-thawing, detaching, and drying. 


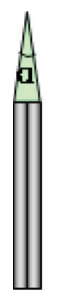

A

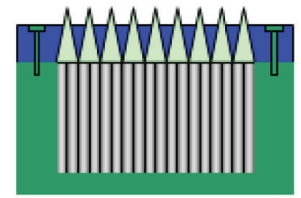

B

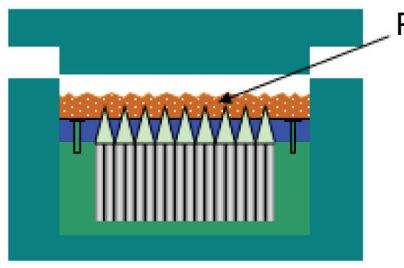

C
Purple sand mud

\section{1}

Figure 2 Fabrication of male mold of microneedles: (A) a single finished hexagonal steel stick; (B) thousands of steel sticks fixed into a steel bucket to form the core of the male mold; (C) the male mold with purple sand spreading smoothly; (D) the female mold prepared using the male mold.

(SX-5-12; Botai Experimental Equipments, Shanghai, China) to remove the remaining water at a temperature of $300^{\circ} \mathrm{C}$ for 2 hours. The final female mold product was obtained after calcinating it at a temperature of $920^{\circ} \mathrm{C}-1040^{\circ} \mathrm{C}$ for 3 hours and cooling it to room temperature (Figure 2D).

\section{Preparation of the microneedle patches}

Two types of microneedles were fabricated to meet different therapeutic needs. For sustained release, the microneedle was prepared as a whole patch, with the drug loaded both into the needles and a backing layer. For fast release, the drug was simply loaded into the needles.

The polymer solution used to fabricate the microneedle patch was a mixture of PVA, dextran, and CMC. The polymer powders were slowly added to water in a wide-mouth bottle with magnetic stirring to form a $20 \%$ solution. After it was sealed with silver paper, the solution was heated to boiling and cooled to room temperature to form a hydrophilic polymer solution. Insulin was added to the solution directly, and then the mixture was stirred for 2 hours to make sure the drug was well dispersed. For sustained-release microneedles, the solution was cast in the female mold and pulled into microholes with the help of a vacuum on the opposite side. The mold with the polymer solution was frozen in a $-20^{\circ} \mathrm{C}$ refrigerator for 2 hours and then thawed at $4^{\circ} \mathrm{C}$ for 1 hour. This freeze-thaw cycle was repeated twice (three cycles in all) to cross-link and solidify the polymer. A piece of undried microneedle patch was prepared after detaching it from the female mold.

For fast-acting microneedles, a blank backing membrane was prepared. PVA and dextran powders were mixed (98:2) and added into a round-bottom flask with water to form a $36 \%$ polymer solution. This solution was sealed and heated to boiling with power-driven simultaneous stirring. When cooled to about $60^{\circ} \mathrm{C}$, the solution was poured onto a piece of quadrate glass board on the experimental table, followed by covering and pressing smoothly with another identical glass board to form the polymer membrane.
The membrane's thickness was controlled by adjusting the height of the supporters ( $1 \mathrm{~mm}$ was used in this study) at the four angles. After two freeze-thaw cycles, the membrane was cut into a circular shape to form a piece of blank backing layer. After casting and pulling the drug-loaded solution into the female mold, the backing layer was applied to the surface of the mold and pressed smooth. The next step was the same as for the sustained-release microneedles.

\section{Drying}

Both the free-drying step (step 1) and fixed-drying step (step 2) were carried out. In step 1, the microneedle patch was dried and shrunk to meet the size required. Subsequently, in step 2, the patch was fixed in a drying environment (low relative humidity and low pressure) to remove the remainder of the water. A special drying unit was prepared to carry out the drying process. A unit designed to dry one piece of patch was composed of three parts: bottom plate, a press board made of Teflon (press 1), and another press board made of stainless steel (press 2). Press 1 and press 2 were used to carry out step 1 and step 2, respectively (Figure 3 ). The bottom plate was made of a stainless-steel board with a round area removed and replaced by wire netting (Figure 3). The distance between the wire-netting surface and the plate's upper surface was about $0.9 \mathrm{~mm}$. Press 2 had the same shape as press 1 , except for a welding ring $(1.0 \times 0.5 \mathrm{~mm})$ used to fix the microneedle patch (Figure 3 ). Drying units can be integrated in a big plate, as in this study. We prepared each drying board with six drying units.

The undried microneedle patch was placed on the wire netting of a unit, with needles facing upward. After it was covered with press 1 , the patch dried and contracted freely in still or flowing air. When it reached the desired size, the patch was fixed by press 2 and transferred into a vacuumdrying oven for 12 hours. To investigate the effect of relative humidity and airflow rate in the drying process, microneedle patches were dried in environments with humidity of $50 \%$ 


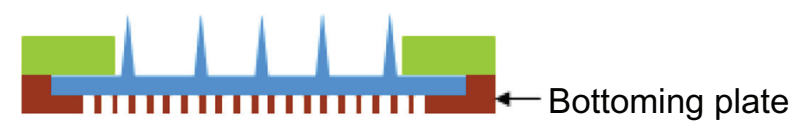

Free drying

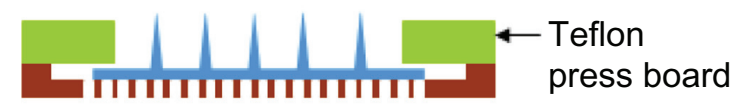

Fixed drying

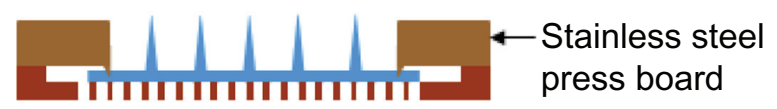

Final patch

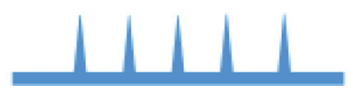

Figure 3 Drying process of microneedle patch. In the first step, the patch was dried with shrinking to adjust size and distance of needles. In the following step, the patch was completely dried with its size fixed.

and $5 \%$ and airflow rates of $0 \mathrm{~m} / \mathrm{second}$ and $1 \mathrm{~m} / \mathrm{second}$, respectively. The final microneedle product was gained by cutting the dried patch into a designated size.

\section{Properties of the microneedles} Microneedle skin insertion

The hardness of the microneedles was determined by skin insertion. A piece of pigskin without subcutaneous fat was shaved and cleaned, and affixed under mild tension to a wooden plate. A microneedle patch with a diameter of $12 \mathrm{~mm}$ was inserted by pressing against the backing layer with a thumb, using a force of approximately $1.5 \mathrm{~N}$, and then removed immediately after the insertion. The microneedle insertion site on the skin surface was exposed for 10 minutes to trypan blue to dye the sites of stratum corneum perforation. After wiping residual dye from the skin surface with dry tissue paper, the skin and its tissue slice were viewed by microscopy (T1-SM; Nikon, Tokyo, Japan).

\section{Swelling of the microneedles}

The PVA microneedles have a property of swelling, which serves to open the diffusion channel for drug release when absorbing body fluid. To test the swelling of the microneedles, six blank patches were inserted into human arms and then covered with rigid tape (9860; 3M, St Paul, MN). The patches were detached from the skin and observed immediately at times of $1,2,3,4,5$, and 6 hours.

\section{In vitro release of insulin}

The release kinetics of insulin from the fast-acting microneedle patches $(n=6)$ were examined by applying a patch to a piece of skin mounted in a Franz cell at $37^{\circ} \mathrm{C}$. The microneedle patch was pressed onto the skin using the $3 \mathrm{M}$ baking membrane. Insulin released into the receiving medium (pH 7.4 phosphate-buffered saline) in the Franz cell was determined using a Shimadzu (Kyoto, Japan) HPLC system equipped with a C-18 column $(4.6 \times 250 \mathrm{~mm}$; Agilent Technologies, Santa Clara, CA). The receiving medium was replaced by a fresh medium and subjected to HPLC measurement at $0.5,1,2,3,4$, and 6 hours after release. Finally, the patch examined for release kinetics was cut into small pieces and suspended in the same releasing buffer at $37^{\circ} \mathrm{C}$ to collect the insulin remaining in the patch in order to assess the total insulin loading. The cumulative fractional release of insulin from the microneedle patches was calculated by dividing the measured amount at each sampling time by the total loaded insulin.

\section{Results and discussion Preparation of the female mold}

The purple-sand mold's breathability varied with different calcinating temperatures. As shown in Table 1, as the temperature increased, the mold became darker in color (Figure 4) and its breathability declined. The siphonage was serious with temperatures lower than $940^{\circ} \mathrm{C}$ (Table 1 and Figure 4A). The optimized temperature range for the female mold was $960^{\circ} \mathrm{C}-1000^{\circ} \mathrm{C}$, in which the mold possessed good breathability and less siphonage.

The calcinating time was also important for properties of the female mold. Seven groups of adobes were calcinated in $960^{\circ} \mathrm{C}$ for $1,2,3,4,6,8$, and 12 hours, respectively (Table 2 ). The ideal period for the female mold was 3-6 hours, and 3 hours was commonly used to save time. As shown in Tables 1 and 2, the shrinkage of the mold (shrinkage-1) was also tested, which was useful in calculating the distribution of microneedles combined with the drying process.

\section{Fabrication of the microneedle patches}

An important step in the fabrication process was preparing the polymer solution. To reach the aim of obtaining a sufficient dosage encapsulated in the needles, insulin dispersed in the polymer solution was in a suspended form because 
Table I Properties of purple-sand female mold calcinated at different temperatures (with calcinating time of 3 hours)

\begin{tabular}{llllllll}
\hline $\begin{array}{l}\text { Temperature } \\
\left({ }^{\circ} \mathrm{C}\right)\end{array}$ & 920 & 940 & 960 & 980 & 1000 & 1020 & 1040 \\
\hline Color $^{\mathrm{a}}$ & + & + & ++ & ++ & ++ & ++ & +++ \\
Breathability $^{\mathrm{b}}$ & +++ & +++ & ++ & ++ & + & + & + \\
Siphonage $^{\mathrm{c}}$ & +++ & +++ & ++ & ++ & ++ & + & + \\
Shrinkage-I (\%) $^{8}$ & 89.6 & 86.9 & 84.8 & 83.6 & 81.7 & 79.8 & 79.5 \\
\hline
\end{tabular}

Notes: ${ }^{\mathrm{a}}+$, yellow; +++ , black. ${ }^{\mathrm{b}}+++$, polymer solution can be pulled into microholes within I minute;,$++ \leq 5$ minutes;,$+>5$ minutes. ${ }^{c}+++$, hydrophilic and diffused; ++ , hydrophilic; +, hydrophobic.

of the drug's poor solubility. Each batch of microneedle patches was fabricated using a fresh drug-loaded polymer solution, each being stirred long enough to ensure uniform dispersion.

However, long stirring of the drug and the polymer solution produced many bubbles, which adversely affected the casting process and needle shape. A vacuum was used to try and eliminate the bubbles, but failed due to the high viscosity of the polymer solution. Moreover, a centrifugal method was also infeasible because of the drug deposition induced by centrifugal force. In our study, a spot of alcohol was added to the mixture, and this solved the problem. Alcohol was an ideal reagent due to its common use in insulin extraction. In addition, the alcohol had no effect on the microneedle patch because it was extracted completely in the drying process.

\section{Drying}

Drying boards designed for microneedle patches are shown in Figure 5. Six drying units were integrated in a steel plate with two protrusions to fix the press board. In the freezedrying step, shrinkage of the microneedle patch (shrinkage-2) was observed. In the static-air condition, the shrinking rate was negatively related to the relative humidity in the air (Figure 6A). Conversely, the drying rate accelerated with increased airflow rate (Figure 6B). Compared to relative humidity, the flowing air provided a better drying rate with
Table 2 Properties of purple-sand female mold calcinated with different times (at $960^{\circ} \mathrm{C}$ )

\begin{tabular}{llllllll}
\hline $\begin{array}{l}\text { Time } \\
\text { (hours) }\end{array}$ & I & $\mathbf{2}$ & $\mathbf{3}$ & $\mathbf{4}$ & $\mathbf{6}$ & $\mathbf{8}$ & $\mathbf{1 2}$ \\
\hline Color $^{\mathrm{a}}$ & + & + & ++ & ++ & +++ & +++ & +++ \\
Breathability $^{\mathrm{b}}$ & +++ & +++ & ++ & ++ & + & + & + \\
Siphonage $^{\mathrm{c}}$ & +++ & +++ & ++ & ++ & ++ & + & + \\
Shrinkage-I (\%) & 86.8 & 85.5 & 84.8 & 84.1 & 82.3 & 80.9 & 80.6 \\
\hline
\end{tabular}

Notes: ${ }^{a}+$, yellow; +++ , black. ${ }^{b}+++$, polymer solution can be pulled into microholes within I minute;,$++ \leq 5$ minutes;,$+>5$ minutes. ${ }^{c}+++$, hydrophilic and diffused; ++ , hydrophilic; +, hydrophobic.

light wind. However, the flowrate of the wind had to be controlled at a certain level since strong winds distorted the microneedle patch.

Shrinking the patches in the drying process provided an important advantage in controlling the needles' intervals. The final intervals of the microneedles were calculated using the following formula: microneedle distance $=$ needle distance of male mold $\times$ shrinkage- $1 \times$ shrinkage- 2 . Shrinkage- 1 was constant when the calcinating parameters of purple sand were fixed. The difficulty level of preparing the male mold was decreased by the microneedle patch's shrinking property. In brief, the flexible-sized hexagonal steel sticks used to form the male mold were selected, thereby reducing the rejection rate and cost. Also, shrinking via drying maintained the needles' shape. The microneedle patch dried using only step 2 would produce needles with larger $\alpha$-values because of water loss in the needle tip but a fixed size in the base. These short and blunt microneedles were ineffective in penetrating the skin's epidermis.

\section{Skin insertion}

With the expectation that the microneedles should be sufficiently hard, we inserted a piece of a microneedle patch with a diameter of $12 \mathrm{~mm}$ into pigskin and found that microneedles ( $0.8 \mathrm{~mm}$ in length and $0.3 \mathrm{~mm}$ in base) were embedded reliably into the skin using a force of about

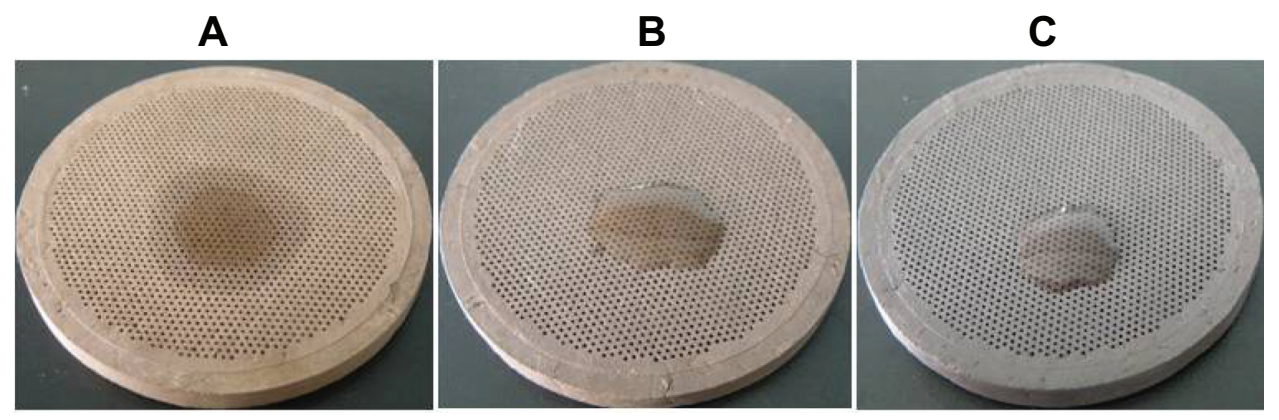

Figure 4 Siphonage of female molds prepared at different temperatures: (A) $940^{\circ} \mathrm{C}$; (B) $980^{\circ} \mathrm{C}$; (C) $1020^{\circ} \mathrm{C}$. 

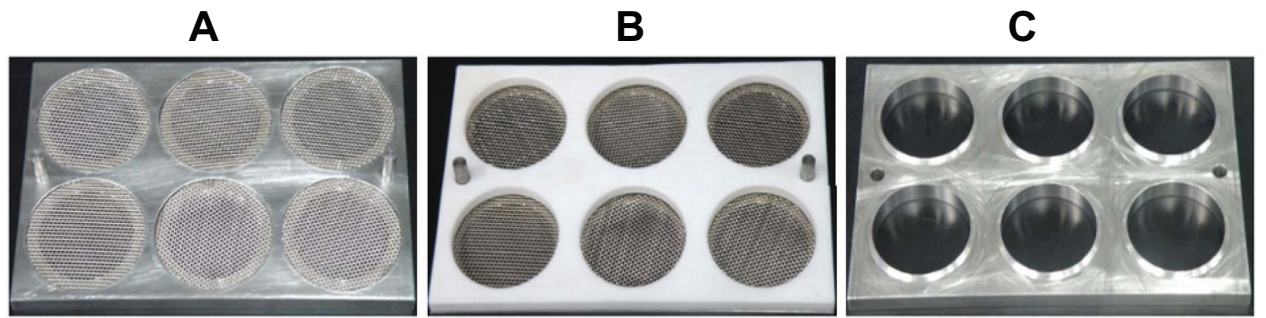

Figure $\mathbf{5}$ Instruments used in microneedle-patch drying: (A) stainless plate with six drying units; (B) Teflon board (press I) over the drying plate; (C) press 2 made of stainless steel with enough weight to fix microneedle patch.

A

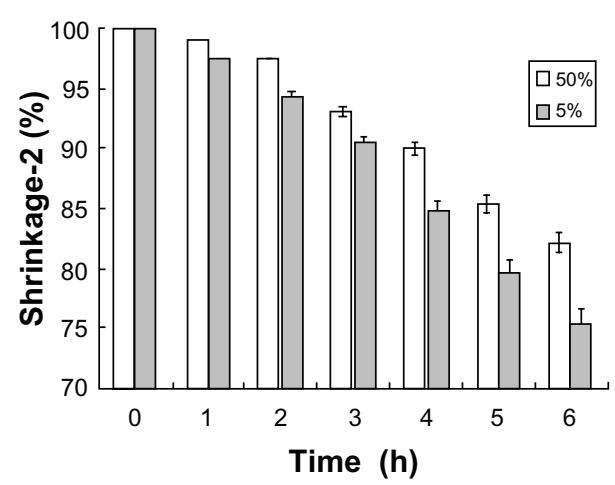

B

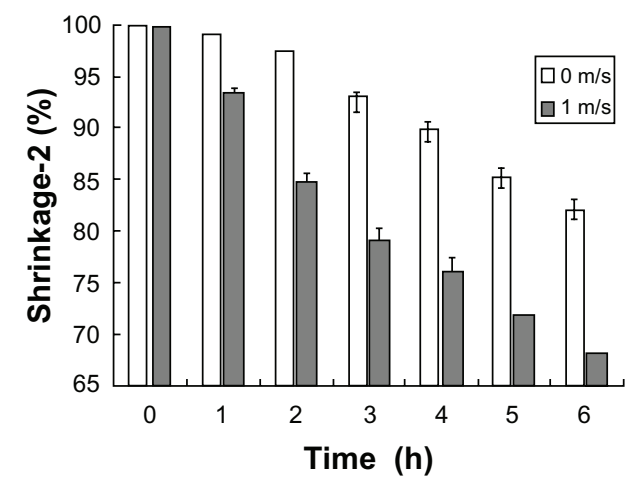

Figure 6 Shrinkage of microneedle patch in free-drying process: $(\mathbf{A})$ effect of relative air humidity; (B) effect of airflow rate.

A
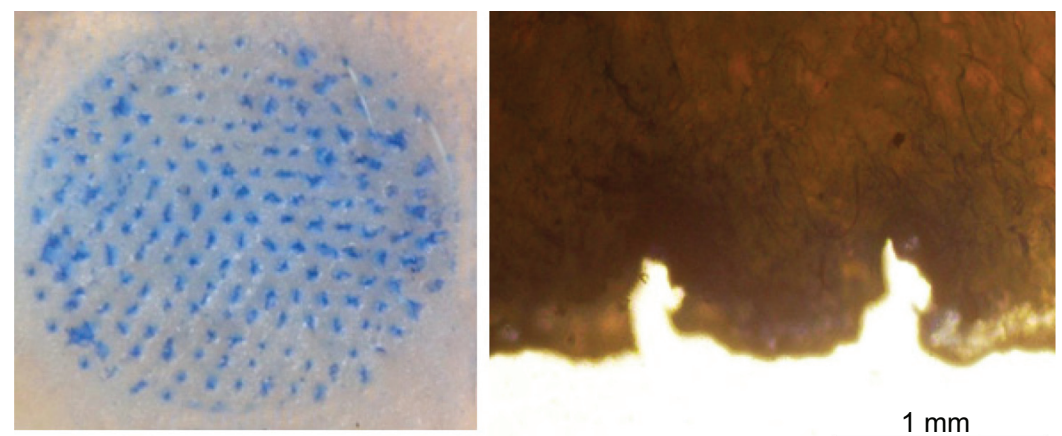

$1 \mathrm{~mm}$

Figure 7 Skin penetration by microneedles: (A) pigskin dyed with trypan blue after microneedle patch removed; (B) micrograph of tissue slice of the pigskin.
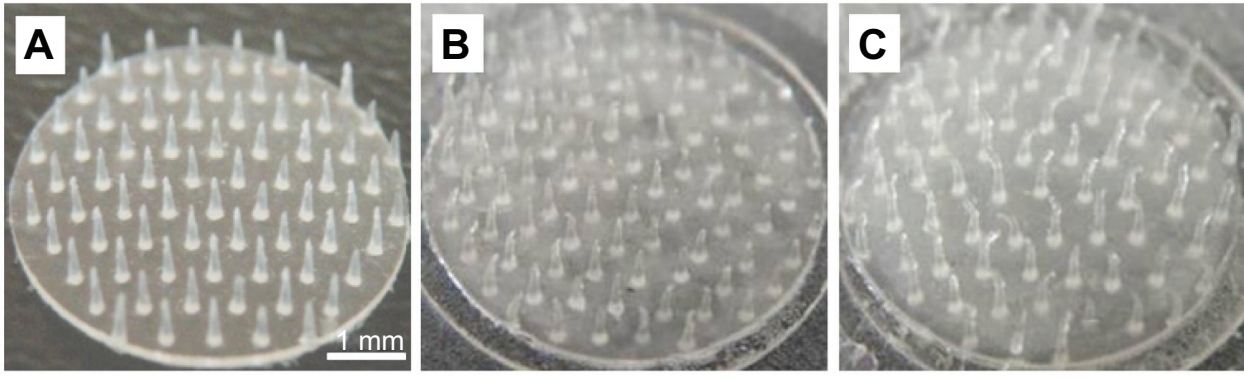

Figure 8 Swelling of microneedles after inserting into human skin: (A) before patching; (B) I hour after patching; (C) 3 hours after patching. 
$5 \mathrm{~N}$ with one thumb. After treating the skin with a dye that selectively marks sites of skin penetration, we found that all of the microneedles in the patch inserted into the skin (Figure 7A). A histological test of skin pierced with microneedles showed penetration depths of approximately $0.4 \mathrm{~mm}$ (Figure 7B), which was about half of the length of a microneedle. This can be explained by the deformation of the skin's surface known to occur during microneedle insertion due to the skin's viscoelasticity. ${ }^{16}$ The relatively wide base $(300 \mathrm{~mm})$ and large $\alpha$-value of the needle tip contributed to this incomplete insertion. Further optimization of the microneedle's shape, such as $\alpha$-value, shrinkage- 2 , and needle distance, may increase the insertion depth. In addition, formulation factors and the preparation process can also be considered to improve the needles' strength. For example, increasing the dextran concentration in the polymer solution and freeze-thaw cycles were effective methods to strengthen microneedles. However, as discussed below, partial microneedle insertion may be adequate for the swelling property presented in this study.

\section{Swelling}

Compared to other hydrophilic microneedles, ${ }^{8,11}$ the PVA microneedle would not dissolve in the skin, but swelling and therefore diffusion channels were opened for drug release. ${ }^{17}$ A sustained type of microneedle patch was prepared to test swelling. The results are shown in Figure 8, in which the needles were adequately swollen after embedding into human skin. The weight of the microneedle patch varied from $40 \mathrm{mg}$ to about $55 \mathrm{mg}$, and the swelling could be adjusted by adding some polymer additives. Take $\mathrm{CMC}$, for example: when absorbing fluid, $\mathrm{CMC}$ dissociated into long chains with a negative charge, which were mutually exclusive and produced a large space to hold water. Other polymers such as dextran and polyvinylpyrrolidone were diluents to crosslinking points. The polymer additives were effective in formulation to control the drug-release rate, both for sustained microneedles and fast-acting microneedles.

\section{In vitro release of insulin}

To examine the release kinetics of insulin from PVA microneedle patches, the dry patches were applied to porcine skin that was mounted on Franz cells filled with buffer solution. As shown in Figure 9, 30\% of the total insulin load was released within the initial 30 minutes, followed by an additional $26 \%$ of the loaded cargo being released within 4 hours of patching. Although the kinetics of insulin

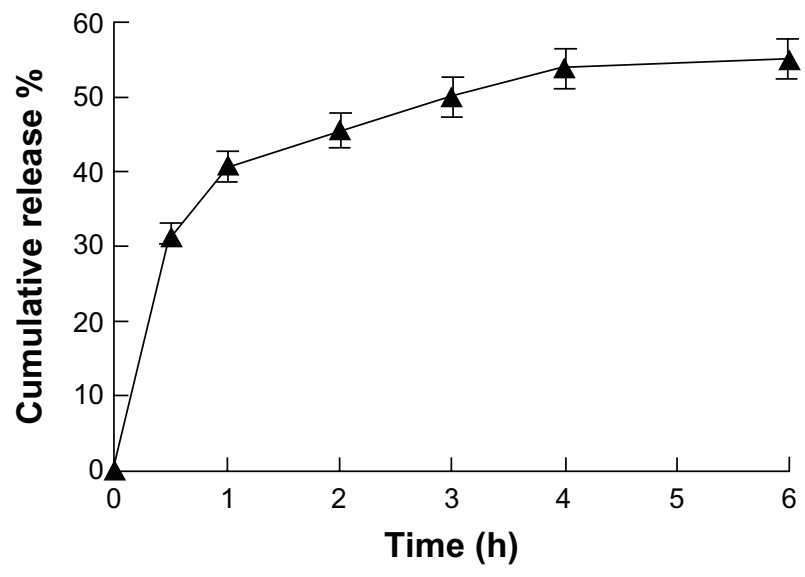

Figure 9 In vitro release profile of insulin from microneedle patches to Franz cells $(n=6)$.

release to a Franz cell may not necessarily be the same as the release kinetics to the dermis layer, the rapid insulin release (Figure 9) to human skin should be sufficient. The diffusion resistance from dermis to subcutaneous tissue may be a concern for practical usage of this insulin patch. However, both the force-assisted hollow microneedle patches and the body fluid-triggered soluble microneedle patches have been found to be capable of offering comparable insulin uptake to subcutaneous injections. ${ }^{2,11}$ The insulin-release rate shown in Figure 9 is comparable to those from the two microneedle patches above. The microneedle system could also improve through further formulation optimization.

\section{Conclusion}

The purple-sand female mold was prepared to fabricate a PVA microneedle patch in this study. This mold had the advantage of being breathable but water impermeable, and in coordination with a vacuum on the opposite side, it perfectly met the demand of hydrophilic microneedle patch preparation. Moreover, in the process of drying the crosslinked microneedle patch, we developed instruments for free drying and shrinking, which controlled the needle distance and thereby simplified the preparation of the male mold.

We fabricated microneedles by the physical method of freeze-thawing them in a solidified polymer solution. This process was simple and safe compared with chemical cross-linking, in which a cross-linking agent is added. ${ }^{11,18}$ An insulin-loaded microneedle patch was prepared and characterized in terms of hardness, swelling, and in vitro release. The pharmacokinetics and pharmacodynamics are now ongoing, and will be reported in future. 


\section{Disclosure}

The authors declare no conflicts of interest, financial or otherwise, in this work.

\section{References}

1. Henry S, McAllister DV, Allen MG, Prausnitz MR. Microfabricated microneedles: a novel approach to transdermal drug delivery. $J$ Pharm Sci. 1998;87(8):922-925.

2. Prausnitz MR. Microneedles for transdermal drug delivery. Adv Drug Deliv Rev. 2004;56(5):581-587.

3. Bartus RT, Tracy MA, Emerich DF, Zale SE. Sustained delivery of proteins for novel therapeutic products. Science. 1998;281(5380):1161-1162.

4. Wilke N, Mulcahy A, Ye SR, Morrissey A. Process optimization and characterization of silicon microneedles fabricated by wet etch technology. Microelectron J. 2005;36(7):650-656.

5. Chabri F, Bouris K, Jones T, et al. Microfabricated silicon microneedles for nonviral cutaneous gene delivery. Br J Dermatol. 2004;150(5): 869-877.

6. Shikida M, Hasada T, Sato K. Fabrication of a hollow needle structure by dicing, wet etching and metal deposition. J Micromech Microeng. 2006; 16:2230-2239.

7. Wang PM, Cornwell M, Hill J, Prausnitz MR. Precise microinjection into skin using hollow microneedles. J Invest Dermatol. 2006;126(5): 1080-1087.

8. Lee JW, Park JH, Prausnitz MR. Dissolving microneedles for transdermal drug delivery. Biomaterials. 2008;29(13):2113-2124.
9. Miyano T, Tobinaga Y, Kanno T, et al. Sugar micro needles as transdermic drug delivery system. Biomed Microdevices. 2005;7(3): 185-188.

10. Sullivan SP, Murthy N, Prausnitz MR. Minimally invasive protein delivery with rapidly dissolving polymer microneedles. Adv Mater. 2008;20(5):933-938.

11. Sullivan SP, Koutsonanos DG, del Pilar Martin M, et al. Dissolving polymer microneedle patches for influenza vaccination. Nat Med. 2010;16(8):915-920.

12. Park JH, Allen MG, Prausnitz MR. Polymer microneedles for controlled-release drug delivery. Pharm Res. 2006;23(5):1008-1019.

13. Kuo SC, Chou YA. Novel polymer microneedle arrays and PDMS micromolding. Tamkang J Sci Eng. 2004;7(2):95-98.

14. Ting GU. Cultural implications of zisha pottery teapot. China Ceramic Industry. Mar 2011. Available from: http://en.cnki.com.cn/Article_en/ CJFDTOTAL-ZTCG201103016.htm. Accessed February 20, 2012.

15. Yixing.com [website]. Are Yixing teapots healthy and safe? Available from: http://www.yixing.com/teapot_info.html?page=faq. Accessed February 20, 2012.

16. Martanto W, Moore JS, Couse T, Prausnitz MR. Mechanism of fluid infusion during microneedle insertion and retraction. J Control Release. 2006;112(3):357-361.

17. Jin T, inventor. Phase-transition polymeric microneedles: World Intellectual Property Organization patent WO/2010/040271. Apr 15, 2010.

18. Woolfson AD, Morrow DIJ, Morrissey A, Donnelly RF, McCarron PA, inventors. Delivery device and method. World Intellectual Property Organization patent WO/2009/040548. Apr 2, 2009.
International Journal of Nanomedicine

\section{Publish your work in this journal}

The International Journal of Nanomedicine is an international, peerreviewed journal focusing on the application of nanotechnology in diagnostics, therapeutics, and drug delivery systems throughout the biomedical field. This journal is indexed on PubMed Central, MedLine, CAS, SciSearch ${ }^{\circledR}$, Current Contents ${ }^{\circledR} /$ Clinical Medicine,

\section{Dovepress}

Journal Citation Reports/Science Edition, EMBase, Scopus and the Elsevier Bibliographic databases. The manuscript management system is completely online and includes a very quick and fair peer-review system, which is all easy to use. Visit http://www.dovepress.com/ testimonials.php to read real quotes from published authors. 